\title{
Protée
}

\section{Aby Warburg et les timbres en tant que document culturel}

\section{Karen Michels et Charlotte Schoell-Glass}

Volume 30, numéro 2, automne 2002

Sémiologie et herméneutique du timbre-poste

URI : https://id.erudit.org/iderudit/006734ar

DOI : https://doi.org/10.7202/006734ar

Aller au sommaire du numéro

Éditeur(s)

Département des arts et lettres - Université du Québec à Chicoutimi

ISSN

0300-3523 (imprimé)

1708-2307 (numérique)

Découvrir la revue

Citer cet article

Michels, K. \& Schoell-Glass, C. (2002). Aby Warburg et les timbres en tant que document culturel. Protée, 30(2), 85-92. https://doi.org/10.7202/006734ar

\section{Résumé de l'article}

L'historien de l'art allemand Aby Warburg (1866-1929), fondateur de la Kulturwissenschaftliche Bibliothek Warburg à Hambourg (1926) - rétablie à Londres en 1933 sous le nom de l'Institut Warburg -, est célèbre surtout pour son approche originale des études culturelles. Spécialiste de l'art et de la culture de la Renaissance, il a transformé la conception de l'histoire de l'art de son temps en éliminant autant que possible la barrière entre les formes classique et populaire de l'art. Pour Warburg, une forme populaire, telle que le timbre-poste, était en principe aussi riche en tant que message culturel que l'œuvre d'art classique et devait jouer un rôle positif dans l'expression culturelle. Ainsi voulait-il, dans les années 1920, participer à la création d'une émission de timbres commémorant le traité de Locarno, le premier accord de coopération entre la France, la Grande-Bretagne et l'Allemagne, signé après la Première Guerre mondiale. Cet article suivra le processus de conception et de réalisation de ce timbre, prévu pour la poste aérienne et portant l'inscription "Idea Vincit ", en soulignant l'importance, dans la République Weimar, de la création dans le domaine public de formes modernes de design.
Ce document est protégé par la loi sur le droit d'auteur. L'utilisation des services d’Érudit (y compris la reproduction) est assujettie à sa politique d'utilisation que vous pouvez consulter en ligne.

https://apropos.erudit.org/fr/usagers/politique-dutilisation/ 


\section{ABY WARBURG ET LES TIMBRES EN TANT QUE DOCUMENT CULTUREL}

Karen Michels et Charlotte Schoell-Glass

L'œuvre de l'historien de l'art hambourgeois Aby Warburg (1866-1929) a eu un destin singulier. Elle ne faisait pas partie du mainstream de l'histoire de l'art et n'a que plus tard influencé cette discipline. Le nom de Warburg continue de vivre dans l'institution fondée à Hambourg et émigrée à Londres, le Warburg Institute. Encore aujourd'hui, l'œuvre de Warburg est de la plus grande actualité, mais en même temps une pierre d'achoppement irritante quoique productive. Depuis 1970, sa «kunstgeschichtliche Kulturwissenschaft» (science de la culture traitant de l'histoire de l'art) est étudiée en Italie, en Allemagne, en France et aux États-Unis avec un intérêt toujours grandissant, elle est traduite et prise comme point de départ pour de nouvelles recherches ${ }^{1}$.

Warburg consacra sa vie à la Renaissance, en particulier aux débuts de la Renaissance florentine; sa thèse traitait déjà d'un problème de l'histoire de l'art de Florence, la Vénus et la Primavera de Botticelli. La réception de l'art antique, déjà visible dans ses premiers travaux, le fascinait tout particulièrement et il tentait de la suivre en utilisant des chemins inhabituels. Il constata qu'on devait tout spécialement porter notre attention sur «les accessoires en mouvement» des cheveux et des robes, afin de comprendre la fonction de la réception artistique de l'Antiquité du XVe siècle. Après de longues années d'intensives études d'archives à Florence, il formula son credo: «le mot en image», qu'il opposa à une science de l'art orientée uniquement vers l'analyse de la forme, nommée à cette époque «la méthode prédominante».

En tant que savant, Warburg avait, au début du XXe siècle, fondé à Hambourg une bibliothèque, la «Kulturwissenschaftlichen Bibliothek Warburg» (KBW), qui était surtout consacrée à l'étude de l'art de la Renaissance. Mais le nom de cette bibliothèque révélait un programme dépassant largement le cadre étroit de l'histoire de l'art: il s'agissait avant tout pour Warburg de surmonter le «parti pris de police frontalière» de sa discipline. En effet, sa préoccupation principale était de comprendre les cultures du passé par leurs images et leurs documents ("Actes»). Ce fut bientôt le processus de l'échange artistique, du transport d'images et de symboles, souvent sur plusieurs périodes et sur de longues distances, qu'il cibla d'abord, comme dans ses études, sensationnelles pour l'époque, sur les fresques 
astrologiques de la Sala dei Mesi du Palazzo Schifanoia à Ferrara. Dans les années 1900-1920, Warburg publia de courts travaux, par exemple sur les tapisseries flamandes, ou encore sur les tracts allemands ou les tableaux d'autel hollandais, qui fonctionnaient également comme «véhicule d'images». Ce faisant, Warburg réalisa rapidement, dans le cadre de sa recherche, qu'une séparation entre l'art noble et l'art trivial n'était pas indiquée - il s'agissait là d'une nouvelle conception pour la recherche en histoire de l'art. De même, Warburg remarqua très tôt qu'une distinction selon les disciplines académiques n'était plus valable. Ses recherches étaient orientées vers des problèmes spécifiques et tout ce qui pouvait contribuer à leurs solutions était utilisé. Il prit tout autant au sérieux les sources littéraires que l'astrologie, le commerce, la fête, le théâtre, la danse et les rites; les livres de compte de la Renaissance tout comme les horoscopes arabes et les chroniques familiales florentines. Il porta son regard aussi bien sur des chefs-d'œuvre canonisés des arts plastiques que sur - c'était là tout nouveau -des artéfacts dans le domaine des arts appliqués et de la culture quotidienne: cartes géographiques, affiches publicitaires, descriptions de défilés solennels, tapisseries, photos de presse et timbres. Cette démarche fut à l'origine de l'étude et de l'analyse des timbres en histoire de l'art.

\section{L'HISTORIEN DE L'ART EN TANT QUE PHILATÉLISTE}

"Qu'est-ce que le destin? Il y a une idée en moi sur la signification de l'art philatélique en tant qu'épidiascope de la fête politique au XXe siècle», note Aby Warburg dans son journal de bibliothèque (21 déc. 1926) ${ }^{2}$. Le thème de la réflexion de Warburg est quelque peu énigmatique; pourtant l'originalité des questions posées est déjà manifeste. Premièrement, la création de timbres est, dans son ensemble, interprétée comme un «art». Deuxièmement, il émet la thèse que les timbres reflètent en général les pratiques sociales, comme «la culture de la fête». Au centre des timbres, considérés comme un «art officiel», les événements politiques et sociaux deviennent, grâce à l'«épidiascope», clairement visibles. Les timbres étaient ainsi, pour Warburg, des documents particulièrement expressifs eu égard à leurs fonctions allégoriques vis-à-vis de l'État. Il s'intéressa au timbre en tant qu'insigne de la souveraineté, par rapport aux monnaies et aux gemmes, aux informations que le timbre pouvait fournir sur son époque et son niveau de culture, au rang symbolique qu'il possédait. Ce dernier aspect fut particulièrement important pour Warburg. Pour lui, la conception de timbres devenait un «système de création symbolique» ${ }^{3}$.

Dès 1918, Warburg, lui-même philatéliste, avait proposé à son éditeur B.G. Teubner à Leipzig une publication sur le "développement des timbres en histoire de l'art», mais Teubner refusa en précisant que les acheteurs ne seraient pas assez intéressés par une étude aussi spécifique. Pourtant Warburg garda les timbres en vue. Dans sa bibliothèque, il aménagea une section consacrée à ce thème et étudia les timbres sous l'angle de l'histoire de l'art, ceux dont les motifs pouvaient avant tout s'intégrer à son argumentation consacrée à la preuve d'un processus d'échange culturel. Son intérêt pour les timbres postaux, et en conséquence pour l'iconographie d'un symbole officiel, s'est encore intensifié lorsque la bibliothèque emménagea en 1926 dans un nouveau bâtiment, qui fut par la suite élargi en un institut de recherche semipublic. Dans les écrits publiés par Warburg, ses recherches sur les timbres n'ont trouvé qu'un faible écho, mais elles sont documentées dans le journal de bibliothèque précité de la KBW.

Ce journal a débuté en 1926 lors du déménagement de la bibliothèque dans un nouveau bâtiment. Les notations régulières proviennent de Warburg et de ses deux principaux collaborateurs, Fritz Saxl et Gertrud Bing. Jusqu'à la mort de Warburg, le 26 octobre 1929, le journal comptait neuf volumes. On y fait mention d'achats de timbres, de visiteurs qui furent initiés à la "problématique des timbres", de la littérature philatélique qui fut acquise et classée. Par moments, les discussions allaient assez loin, si bien qu'il est arrivé que trois timbres furent 

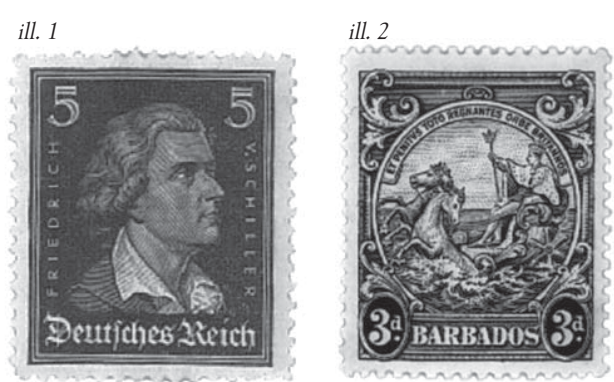

expressément collés dans le journal; ils montraient le Président de la République Paul von Hindenburg, le Président de la République Friedrich Ebert et Johann Wolfgang von Goethe ${ }^{4}$. En outre, Warburg suivait de manière attentive et critique l'émission des nouveaux timbres-poste:

\section{Aivu des exemplaires de timbres (en comparaison avec le nouveau timbre niais). Haas critique devantle parlement (Reichstag) non seulementle timbre de Fredericus mais aussile V.(!!!) de Schiller.5 (ill. 1)}

Il proposa plusieurs fois, avec insistance mais en vain, au Deutschen Museum à Munich, avec lequel il était déjà en pourparlers au sujet de l'installation d'un département d'astrologie, la mise en place d'un département de philatélie. Warburg aurait bien voulu transmettre ses propres recherches à des scientifiques plus jeunes. Ainsi, il nota en décembre 1926: «Ai classé des timbres. Ai porté l'attention de Mlle Sellschopp sur les possibilités d'appliquer l'histoire de l'art au domaine philatélique " ${ }^{6}$. La jeune étudiante hambourgeoise saisit tout aussi peu cette proposition que le fils de Warburg, Max Adolph, qu'il tenta également de passionner pour les timbres. Un des multiples titres, avec lesquels Warburg cherchait à reformuler sans cesse ses thèmes de recherches, était le suivant:

Histoire de l'art des timbres en ébauche. Un essai de Max Adolph Warburg, Dr phil, avec une préface et une étudesurles timbres de la Barbade de Aby Warburg. Édition Idea Victrix. ${ }^{7}$

\section{LA «BARBADE» ET LA «SEMEUSE»}

Warburg s'était donc réservé pour lui-même le travail sur le cas particulier des «timbres de la Barbade». Sur un timbre de la Barbade, île antillaise appartenant à la Grande-Bretagne, il avait découvert la silhouette du roi anglais Edouard VII, qui conduisait, comme jadis Neptune, un char de triomphe tiré par des chevaux de mer (ill. 2) ${ }^{8}$. Il y est écrit: «Il règne sur les mers» («Britannia, rule the waves»). Sur des
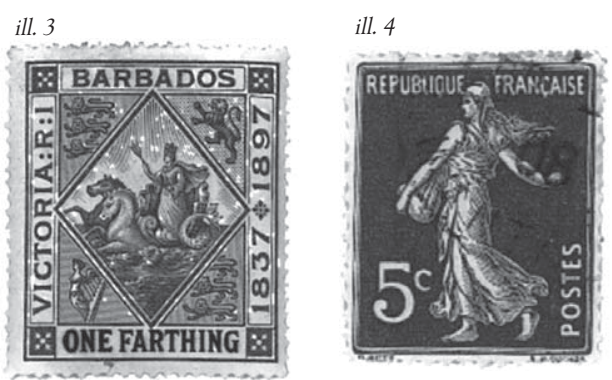

timbres plus anciens, c'est la reine Victoria (ill. 3) qui est représentée dans ce même char triomphal. Le texte, aussi imprimé sur le timbre, est le suivant: «Et penitus toto regnantes orbe Britannos " 9 , une citation transformée - mais de manière décisive - des Bucoliques de Virgile (vers 66): «et penitus toto divisos orbe Britannos». Alors que Virgile caractérise les Anglais comme «entièrement séparés» du reste du monde, la légende du sceau signifie que les Anglais «règnent sur le monde entier». Pour Warburg, c'est l'exemple par excellence d'une «inversion énergétique ${ }^{10}$, d'un renversement de sens. Ce qui servit à réaliser ce motif provenant de la mythologie classique fut un sceau anglais que le roi Charles II avait prêté en 1663 au gouverneur de la Barbade. Warburg qualifie d'acte symbolique cette transformation, effectuée sur le timbre, de Neptune en les traits du souverain anglais et, en même temps, en la figure de Britannia. Edouard VII est ici déguisé de manière mythologique; ainsi, en tant que graphique monochrome, le timbre garde une distance métaphorique avec la réalité. Il fonctionne, selon les mots de Warburg, comme un "symbole énergétique", comme un «inverseur» qui peut transformer l'énergie inhérente à un caractère d'expression. En conséquence, le timbre de la Barbade devint pour Warburg le témoin principal de sa théorie de l'image. Il prouvait non seulement la capacité de survie formelle du caractère de la forme antique, mais aussi la possibilité d'un renouveau de telles formes par des contenus actuels. Warburg n'a pas publié ce matériel, mais il a tout de même présenté les résultats de sa recherche lors de plusieurs conférences ${ }^{11}$.

Warburg correspondait sur cette question avec différents chercheurs, dont E.M. Shilstone à la Barbade, qui a publié l'emblème de l'île antillaise et son iconographie, mais seulement après la mort de Warburg ${ }^{12}$. Sous l'angle historico-culturel se rapportant à l'antiquité, le sceau du XVII siècle sur le timbre apparaît comme un symbole qui s'adapte à 

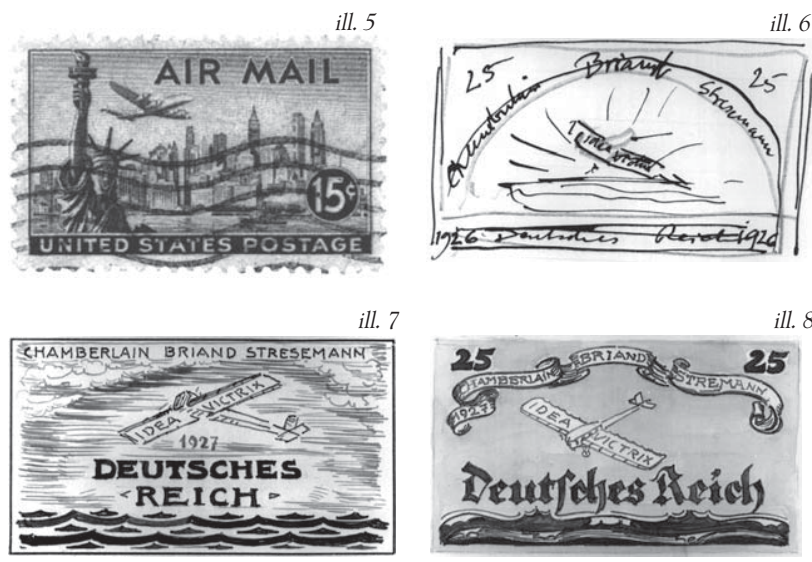

ill. 7

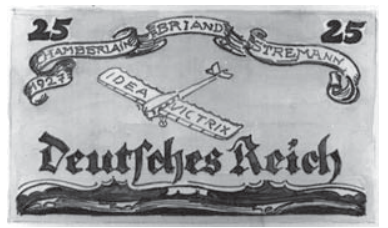

diverses situations, prouvant la viabilité des caractères de l'image antique. Ce qui n'entrait pas dans le cadre des études des savants dans les années 1920 et 1930 est ici la question coloniale, décidément une perspective politique, mais qui a fait récemment l'objet de recherches ${ }^{13}$. Le problème d'une identité nationale, image dont les caractères étaient issus de modèles européens et antiques, y est représenté comme un processus qui se servait d'emblèmes ambivalents. L'image, que les anciennes colonies se faisaient d'elles-mêmes, fut ainsi influencée jusque dans un passé proche.

Un autre exemple nous est offert par un timbre français, la "Semeuse» (ill. 4). Tout comme pour le timbre de la Barbade, Warburg l'a ajouté à une planche dans son "Bilderatlas", atlas d'images ${ }^{14}$. Il représente une jeune femme enveloppée dans un long habit qui caresse légèrement ses chevilles. Dans un mouvement ample, elle tient dans la main gauche un semoir, dans lequel elle puise de la main droite des graines qu'elle épand. Warburg la met en relation avec un enchaînement de motifs, qui documente la continuation de l'antiquité jusque dans les insignes de la souveraineté de sa propre époque. Cet enchaînement a pour point de départ la figure de la ménade antique. Cette image de la femme, représentée le plus souvent dans une danse extatique, se transformait, ainsi le démontrait-il, jusqu'à la renaissance de la forme d'une femme dansant avec légèreté, représentée comme nymphe ou servante dans les œuvres de Botticelli ou de Ghirlandajo, incarnant sans cesse une «mobilité antique idéelle» ${ }^{15}$. Sur le timbre français, la nymphe fait figure de

[...] symbole vénérable de la fécondité de la France aux yeux du public. Sûrement, estelle, au sens pratique, une déesse serviable; même si elle n'apporte pas de foie gras, elle doit, au service du motailé, porter la pensée jusqu'à l'endroitoù la Poste, en d'autres termes, le peuple, tributaire seulement de l'État, le veut. ${ }^{16}$

La «filiation iconographique " 17 n'est donc qu'une partie de l'analyse des timbres; d'un autre côté, la fonction et la perception d'un motif transmis constituent tout autant, pour l'historien de l'art, un élément de réflexion: la ménade, figure et parabole de la passion, se suffisant à elle-même, devient sur le timbre une servante bien réelle de la poste. Pourtant, lorsque Warburg voulut de son côté faire ébaucher un timbre postal aérien au service d'idées élevées, il perdit de vue le lien avec le contexte des signes et des symboles. Il espérait toutefois donner une forme à un événement historique grâce à un caractère d'image fait sur mesure et définitif (ill.5).

\section{«IDEA VINCIT»}

«Le timbre postal aérien met la dynamique d'échange énergétique à la place du transfert de volonté politique» ${ }^{18}$. Le potentiel de médiation du timbre parut d'une telle évidence au savant qu'il alla même jusqu'à utiliser le médium pour ses propres fins afin de répandre une de ses idées politiques favorites. Quand, en 1926, les trois ministres des Affaires étrangères européens, Aristide Briand, Joseph Austen Chamberlain et Gustav Stresemann, reçurent le prix Nobel de la paix pour leurs efforts de négociations lors des Accords de Locarno, Warburg conçut l'idée d'un nouveau timbre postal aérien. Il devait porter au monde entier le message d'une Allemagne de nouveau prête à la communication et à la collaboration internationales et, en même temps, il devait satisfaire à son exigence en matière de symboles de pouvoir et d'expression contemporains. Il commanda l'ébauche (ill. 6) à un vieil ami, l'artiste munichois Alexander Liebmann. Il le pria

[...] de[lui] dessiner d'abord un nouveau timbre allemand en format oblong, qui devait montrer la mer etau-dessus l'avion en plein décollage, portantl'inscription Briand Chamberlain Stresemann. [Il reçut] comme réponse trois échantillons banals 
mais rapidement exécutés. Les propositions d'amélioration ne donnèrent rien (l'Empire allemand dans l'eau). 19

Warburg profita de la visite de Stresemann à Hambourg pour lui présenter cette ébauche de timbre et le conduire à la KBW. Stresemann, républicain par raison, avait trouvé la force de projeter la république de Weimar dans un avenir européen. Le nouvel ordre de paix européen, que les Accords de Locarno devaient fonder, était «l'idée victorieuse» que Warburg voulait ériger en monument commémoratif.

Locarno représentait un espoir etc'estainsique Chamberlain pouvait affirmer qu'iln'yaurait désormais ni vainqueurs ni vaincus. C'est dans ces mêmes termes que Stresemann affirma, lors de la signature des accords le 1er décembre 1925, qu'ilétait appropriéde parlerd'une «idée européenne victorieuse». ${ }^{20}$

Les ébauches d'Alexander Liebmann d'après les esquisses d'Aby Warburg montrent un avion qui s'élève de la droite vers la gauche au-dessus de la surface stylisée de la mer (ill.6). On a expérimenté ce motif en plusieurs variations (ill. 7), mais avant tout avec la typographie (ill.8) pour les légendes "Chamberlain Briand Stresemann", «Deutsches Reich» et "Idea Victrix» ${ }^{21}$. Les ébauches s'inspiraient
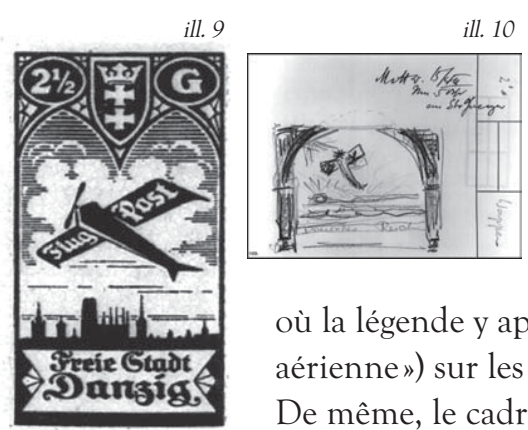
clairement des timbres, déjà en circulation, de la Freistadt Danzig (ill.9), qui montraient un avion au-dessus du schéma de la ville et où la légende y apparaissait déjà («Poste aérienne») sur les ailes de l'avion postal. De même, le cadre aux contours d'arcades dans l'esquisse de Warburg a déjà été ulilisé antérieurement (ill. 10). Les propositions vieux jeu de Liebmann, et à l'évidence aussi sa propre fantaisie d'image, n'étaient pas, aux yeux de Warburg, à la hauteur de l'importance de la rencontre: l'idée victorieuse devait se montrer immédiatement sous une forme moderne orientée vers l'avenir.

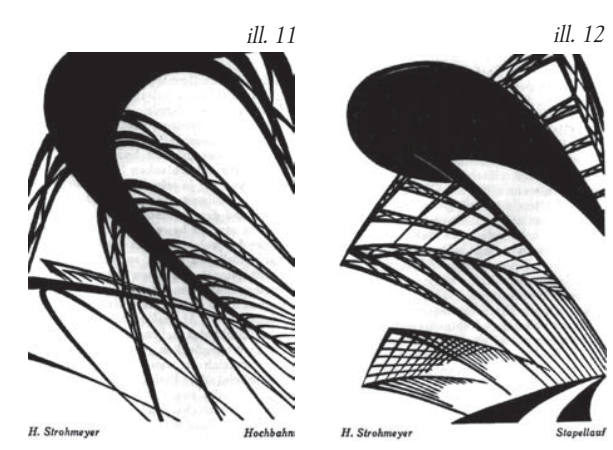

Alors [...] j'eus l'idée que Strohmeyer devait être l'artiste tant recherché, dontj'avais toujours fortementappréciél'élément statique, complété de traits à la fois fantastiques et faisant référence au réel, élément qui m'avait toujours paru exceptionnel. Maisje restais seul avec mon enthousiasme. [...] Jelui montrai mes esquisses minables, non plus pour un timbre mais pour une feuille: vol ascendant. Légende sur la partie inférieure des ailes, et des barres de fer d'un arc qui n'atteignaient leur hauteur qu'en dehors de la surface de la feuille. [...] L'effet de cette feuille sur Stresemann ne se fit pas attendre. 22

La «feuille de lino» d'Otto Heinrich Strohmeyer fut imprimée en 46 exemplaires et remise à Stresemann, Briand et Chamberlain avec l'aide du ministère des Affaires étrangères. En même temps, elle fut envoyée dans le monde entier, comme il est minutieusement documenté dans le journal et noté dans sa correspondance ${ }^{23}$. Son inventeur était un jeune architecte et graphiste ${ }^{24}$ qui avait étudié à Munich. Il quitta cette ville en 1925, «dégoûté par la peste fasciste qui commençait à se faire remarquer par sa brutalité» 25 . À Hambourg et sous la direction de l'architecte Fritz Höger, il avait participé à la construction de la Chilehause, un chef-d'œuvre de l'architecture expressionniste. À cette époque, une série de graphiques vit le jour, lesquels reçurent, au moins sur la scène artistique locale, un accueil favorable: en 1927, «les abstractions hambourgeoises» de Strohmeyer - une suite de sept gravures sur linoléum - ont été commentées dans Der Kreis. Magazin für künstlerische Kultur ${ }^{26}$. L'auteur de cet article tout à fait positif fut le jeune historien de l'art munichois Alfred Neumeyer, qui venait de terminer sa formation par un stage à la Hamburger Kunsthalle et à la K.B.W. Neumeyer fut particulièrement impressionné par le fait que Strohmeyer avait réussi à 
montrer, à partir des motifs représentés - bâtiments techniques comme des ponts, des tunnels ou des bateaux (ill. 11 et 12) -, et en allant au-delà de l'élément constructif, «la tendance intérieure» de l'architecture. En effet, l'artiste avait développé un ensemble de lignes particulièrement dynamique, qui traitait l'objet comme un ornement et projetait en avant son impulsion du plus profond de l'espace de l'image, pénétrant par là même immédiatement la sphère du contemplateur. Il est en outre impressionnant de voir que Strohmeyer a réussi dans ses ébauches à formuler les excès de l'expressionnisme moderne.

Jusqu'à aujourd'hui, aucun exemplaire de ce graphique ne s'est trouvé dans les archives du Warburg Institute ${ }^{27}$. Pourtant, la feuille graphique put être identifiée (par K. Michels) sur une photographie (ill.13), qui montre Warburg, Gertrud Bing et le secrétaire Hans Volmer dans une chambre d'hôtel à

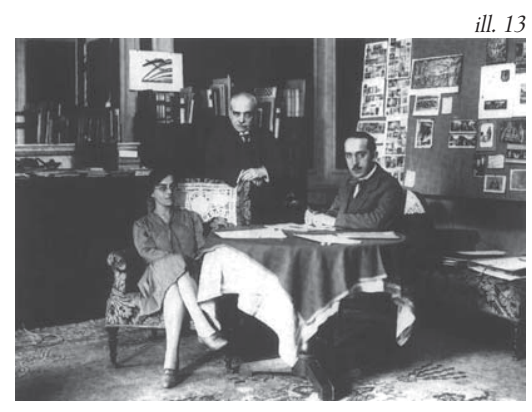

Rome (en 1929), au milieu des planches pour l'atlas d'images (Mnemosyne) en préparation. Cette feuille est posée contre le mur, sur des livres, entre Bing et Warburg, et montre l'avion s'envolant d'un hangar dynamiquement stylisé, allant à l'encontre du contemplateur et portant sous ses ailes fines la

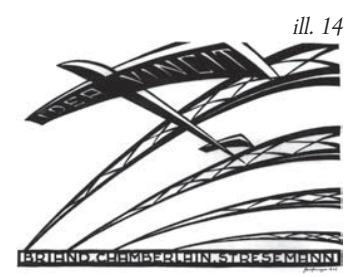

légende «Idea Vincit»; la lisière en bas de l'image porte les noms «Briand Chamberlain Stresemann» (ill. 14).

Le fait que l'«Idea Vincit» fut emportée lors d'un long voyage d'études à Rome prouve que

l'«incarnation" de l'idée européenne de Strohmeyer conservait aussi là-bas sa valeur pour les membres de la KBW. Et cela non seulement, comme il ressort des inscriptions dans le journal de 1926, parce que les participants se tenaient en politique invariablement du côté de l'entente internationale et d'une Europe en paix, mais aussi pour des raisons qui sont à chercher du côté de l'histoire de l'art, comme Warburg l'ambitionnait ${ }^{28}$ :

Il semble que je montrerai à Stresemann la valeur de la reconnaissance de l'ambivalence imagée dans le cercle du monde officiel des images des timbres: par exemple, remplacementde la monumentalité rétrospective antique paruneautodynamique humaine. ${ }^{29}$

Quand Stresemann formule, lors de son dernier discours - devenu célèbre - devant la fédération des peuples, la question: «Et où se trouve la monnaie européenne, le timbre européen? ", il est permis de supposer que ces réflexions remontent aux efforts intensifs de l'historien de l'art Aby Warburg.

\section{AVION ET AÉRONEF}

Aux yeux de Warburg, «l'autodynamique humaine» du Moderne est incarnée par la dynamique technique de l'avion. Son intérêt pour l'aviation s'était déjà manifesté en 1913 dans une étude sur deux tapisseries de Bourgogne, datant du XVe siècle ${ }^{30}$. Sur l'une des tapisseries est représentée la navigation aérienne légendaire d'Alexandre le Grand, qui s'élève dans les cieux dans une cage métallique tirée par quatre rapaces. Warburg était à même de prouver qu'il s'agissait du duc de Bourgogne Charles le Téméraire, qui apparaissait ici sous les traits d'Alexandre le Grand. Mais, en même temps, Warburg interprétait la scène à la lumière de son époque; pour lui, elle montrait que non seulement l'art italien mais aussi l'art nordique de la Renaissance «avaient contribué à 
la tendance de l'homme moderne à dominer le monde». "L'aéronaute moderne, qui se penche sur "l'actuel" problème du refroidisseur du moteur", devrait être informé sur le fait que

[...] son arbre généalogique spirituel, en passant par Charles le

Téméraire-occupéà rafraîchir avec des éponges mouillées les griffes brûlantes de ses rapaces s'élançant vers le haut, rejoint "Alexandrele Grand». 31

Warburg a en outre, comme en témoigne le journal, suivi avec grand intérêt les performances de l'aéronautique moderne, et il les a mises dans un contexte symbolique. Il n'était pas le seul. L'avion est, surtout dans les années 1920, un des grands modèles culturels très répandus. Les effets de l'aéronautique moderne, la nouvelle perception de l'espace et de la vitesse qu'elle induit et sa représentation artistique sont maintenant bien connus ${ }^{32}$. Il est aussi porté à notre connaissance que Warburg observait le développement des techniques de communication, tels le téléphone et le télégraphe, avec une certaine critique. Ces techniques abîmaient selon lui le «sentiment des lointains». La «destruction de la distance» pourrait à la longue faire reculer la culture de l'ère des machines en deçà de ce que les «sciences naturelles sorties du Mythe avaient difficilement atteint» - le retour au chaos serait déjà programmé33. Par contre, il a largement utilisé ces nouvelles techniques - par exemple le nouveau bâtiment de la bibliothèque construit en 1926 est équipé de 28 téléphones, d'un poste pneumatique et de plusieurs ascenseurs de livres. Mais l'avion et le zeppelin, considérés comme des «destructeurs de distance par excellence», revêtent une importance capitale dans son système de valeurs. L'avion, en particulier, n'est pas seulement un nouveau moyen de s'approprier le monde, mais aussi un porteur d' «idée», si bien que Warburg y consacre une planche dans sa dernière œuvre, le «Bilderatlas» 34 .

Dans la photographie romaine, l'avion est perçu, aux yeux des scientifiques qui s'adonnent aux questions de la réception de l'Antiquité, comme un emblème moderne, comme un aveu en faveur de la philosophie des Lumières, d'un humanisme de l'action et du progrès. Et ceci en pleine Rome, le centre du fascisme, contre lequel l'«Idea Vincit» devait être déployée de toutes forces en 1926, comme Warburg le nota dans le rapport annuel du journal au sujet d'un timbre-poste italien qui montre le motif des fascistes, les «fasces» romains:

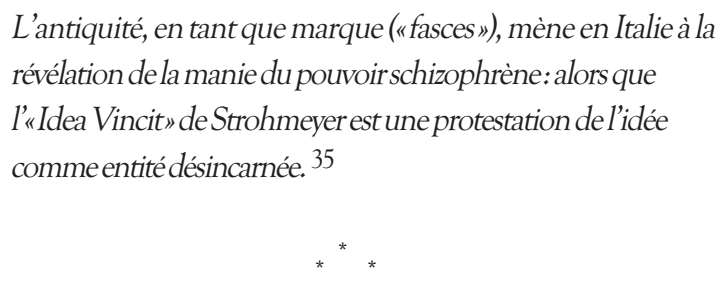

Warburg n'était pas le seul à s'intéresser de manière scientifico-culturelle aux timbres, étude qui évoluait certes dans le cadre de la philatélie, mais qui la devançait aussi: la République de Weimar s'était accordé un «Reichskunstwart», qui était chargé de promouvoir la conception nouvelle et moderne d'emblèmes et d'insignes de la souveraineté du jeune État. Le fonctionnaire en charge du «graphique de l'État» s'appelait Edwin Redslob (1884-1973) ${ }^{36}$. En 1927, lors de l'exposition Le Façonnement artistique du Reich, il vint à Hambourg et fit un discours à la K.B.W. sur le «timbre en tant que document culturel ${ }^{37}$. Il expliqua, entre autres, comme le rapporte le journal quotidien, comment un timbre devait agir et ce qu'il devait produire:

Le timbre devrait être un symbole de la culture de notre pays, un but qui ne peutêtre atteintque par des moyens graphiques. Il ne devrait pas montrer un style de médaille, être une affiche, un paysage ou une image qu' on observe avec lenteur, mais devrait posséderuneapparence de cachetetune empreinte traditionnelle. 38

Dans ces conditions, on ne peut guère s'étonner de ce que la feuille graphique de Strohmeyer, malgré ses qualités formelles pour l'époque, ne fut autrement considérée par Warburg que comme une ébauche pour un timbre-poste. 


\section{NOTES}

1. Voir A. Warburg, La Rinascita del paganesimo antico. Contributi alla storia della cultura, Florence, Raccolti di Gertrud Bing, 1966; A. Warburg, The Renewal of Pagan Antiquity: Contributions to the Cultural History of the European Renaissance, Los Angeles, 1999; A. Warburg, Essais Florentins (prés. d'E. Pinto), Paris, Klincksieck, 1990. P.-A. Michaud, Aby Warburg et l'image en mouvement. suivi de Aby Warburg: Souvenirs d'un voyage en pays Pueblo, 1923; Projet de voyage en Amérique, 1927, Paris, Macula, 1998.

2. A. Warburg, Tagebuch der Kulturwissenschaftlichen Bibliothek Warburg, mit Einträgen von Gertrud Bing und Fritz Saxl, dans K. Michels et C. Schoell-Glass (sous la dir. de), A. Warburg, Gesammelte Schriften. Studienausgabe, vol. 7, Berlin 2001. Dorénavant Tagebuch der KBW.

3. Tagebuch der KBW, p. 62.

4. Ibid., p. 471.

5. Ibid., p. 24.

6. Ibid., p. 33 .

7. Ibid., p. 40.

8. A. Warburg, «Mediceische Feste am Hofe der Valois auf

Flandrischen Teppichen in der Galleria degli Uffizi », dans Gesammelte Schriften, op. cit., p. 256 f.

9. Et les Britanniques régnant complètement sur le monde entier. 10. Tagebuch der KBW, p. 81 .

11. Si Warburg avait suivi le conseil de son collaborateur Fritz Saxl, ce texte aurait été au moins enregistré (voir les archives du Warburg Institute, correspondance, lettre de Fritz Saxl à Warburg, 29 juillet 1928).

12. E. M. Shilstone, "The Society's Corporate Seal", The Journal of the Barbados Museum and Historical Society, 1933-1934.

13. V. Hewitt, "A distant View. Imagery and Imagination in the Paper Currency of the British Empire, 1800-1960", dans E. Gilbert et E. Helleiner (sous la dir. de), Nation-States and Money. The Past, Present and Future of National Currencies, Londres et New York, 1999, p. 97-116, en particulier la page 113 .

14. A. Warburg, Der Bilderatlas Mnemosyne, M. Warnke et C. Brink (sous la dir. de), dans Gesammelte Schriften. Studienausgabe, vol. 2, I, Berlin, 2000.

15. E.H. Gombrich, Aby Warburg. An Intellectual Biography, Londres, The Warburg Institute 1970, p. 105-27.

16. Matériel accompagnant l'exposition «Aby M. Warburg. Mnemosyne", Hamburg, s.d. Texte pour la planche 77: Warburg, conférence à la Chambre de commerce, 1928.

17. Tagebuch der KBW, p. 269 .

18. Ibid., p. 62.

19. Ibid., p. 23.

20. H. Mommsen, Die verspielte Freiheit. Der Wegder Republik von Weimar in den Untergang 1918-1933, Berlin, Propyläen-Verlag, 1989, p. 216.

21. Warburg fit appel à plusieurs experts pour clarifier la question, à savoir comment devait être la version latine correcte. La légende retenue sur les ébauches «Idea Victrix" fut bientôt abandonnée au profit de la forme plus active «Idea Vincit» (Tagebuch der KBW, p. 24). 22. Ibid., p. $23 f$.

23. D. McEwan, «Facetten einer Freundschaft: Aby Warburg und James Loeb. Verwandte, Freunde, Wissenschaftler, Mäzene», dans J. Loeb 1867-1933, Kunstsammler und Mäzen, Schlossmuseum der Stadt Murnau 2000, p. 75-98, p. 88f.

24. Strohmeyer est né en 1895 à Lahr (Forêt Noire), il a étudié l'architecture à la Technische Hochschule de Munich avec T. Fischer; à partir de 1946, il participa à la reconstruction de Hambourg.

25. Voir R. Italiaander, "Gleise und Nebengleise des O.H.

Strohmeyer », Hamburg, Freie Akademie der Künste, 1964, p. 20.

26. A. Neumeyer, «Heinrich Strohmeyers Linolschnitte:

Hamburgische Abstraktionen ", dans Der Kreis, cahier 4, avril 1927,

p. 215-218.

27. Entre-temps, une des feuilles a été retrouvée par D. McEwan,

l'archiviste du Warburg Institute, au Fogg Art Museum de Harvard

University, auquel l'historien de l'art américain P. J. Sachs en avait fait don.

28. Au sujet de Warburg en tant que «bon européen ", voir C. SchoellGlass, «An Episode of Cultural Politics during the Weimar Republic: Aby Warburg and Thomas Mann Exchange a Letter Each ", dans Art History 21, 1998, p. 107-128.

29. Tagebuch der KBW, p.36.

30. "Luftschiff und Tauchboot in der mittelalterlichen

Vorstellungswelt» (1913), dans A. Warburg, Gesammelte Schriften.

Studienausgabe, t. I., Berlin, 1998, p. 241-49, ann. p. 386-388.

31. Ibid., p. 249.

32. Voir C. Asendorf, Super Constellation. Flugzeug und Raumrevolution,

Vienne, Springer, 1997.

33. Ibid., p. 135

34. Der Bilderatlas Mnemosyne, op. cit., planche C,

35. Tagebuch der $K B W$, p. 39.

36. W. Speitkamp, «"Erziehung zur Nation”. Reichskunstwart, Kulturpolitik und Identitätsstiftung im Staat von Weimar", dans H. Berding (sous la dir. de), ; Nationales Bewusstsein und kollektive Identität 2, Frankfort, Suhrkamp, 1994.

37. Hamburger Fremdenblatt, no 226, éd. du soir du 17 août 1927, p. 3. 38. Ibid.

\section{ILLUSTRATIONS}

1. F. Schiller, Deutsches Reich.

2. Barbados, 3 Pence avec Edouard VII.

3. Barbados, One Farthing avec la reine Victoria.

4. République française, 5 centimes.

5. New York, la Statue de la Liberté et un avion, United States Air Mail, 15 cents.

6. Esquisse d'A. Warburg pour «Idea Vincit». The Warburg Institute, archives.

7. A. Liebmann, «Idea Victrix», 1926. The Warburg Institute, archives.

8. A. Liebmann, «Idea Victrix», 1926. The Warburg Institute, archives.

9. Freie Stadt Danzig, Flugpost, 2 1/2 G (19..).

10. Esquisse d'A. Warburg, "Idea Vincit», pour Strohmeyer. The Warburg Institute, archives.

11. H. Strohmeyer, Hochbahn, dans Der Kreis, 1927.

12. H. Strohmeyer, Stapellauf, dans Der Kreis, 1927.

13. G. Bing, A. Warburg et H. Volmer à Rome en 1929, avec la feuille de Strohmeyer. The Warburg Institute, archives.

14. H. Strohmeyer, «Idea Vincit», 1926. The Fogg Art Museum, Harvard University. 